\title{
Caracterización de la flora microbiana y revisión del estado de salud en individuos que laboran en laboratorios de diagnostico.
}

\author{
${ }^{1}$ Silvia E Campuzano F MSc, ${ }^{1}$ Alicia Álvarez de Weldefort y ${ }^{1}$ Judith Camacho K. \\ 1 Programa de Bacteriología, Facultad de Ciencias de la Salud, Universidad Colegio Mayor de Cundinamarca. Bogotá, Colombia. \\ Correspondencia:silvicampu@hotmail.com \\ Recibido:17-08-05 / Aceptado: 10-11-05
}

\begin{abstract}
Resumen
Existen evidencias fundamentadas que indican la validez de los programas de vigilancia epidemiológica para la prevención y control del riesgo biológico y para evitar la contaminación en los ambientes de salud. Debido a que los trabajadores de la salud, son uno de los reservorios de las enfermedades infecciosas, los centros para la prevención y control de las enfermedades de Atlanta, la C.D.C. y otros programas recomiendan, vigilancia, inmunización y adopción de medidas de bioseguridad y programas de control para ciertas enfermedades transmisibles que se dan en ambientes donde se maneja material de salud, representados en fluidos biológicos que corresponden a todas las muestras de los pacientes que deben ser analizadas como la sangre, secreciones purulentas, líquidos orgánicos y los desechos biológicos, considerados como residuos peligrosos; constituidos por gasas, algodones, catéteres y otros.

Uno de los objetivos de estos programas es proteger a quienes laboran con producto biológico contra los factores de riesgo generados y para cumplirlo se debe iniciar con el registro de datos relacionados con la caracterización de la flora microbiana de dichos individuos, para posteriormente diseñar las estrategias pertinentes para el control de la situación. Por consiguiente el presente estudio, aprovecha la investigación para conocer y comparar la microbiota de las estudiantes de bacteriología en contacto permanente con riesgo biológico como resultado de las condiciones de su propio desempeño, relacionado con el manejo continuo de muestras biológicas; frente a estudiantes de trabajo social alejadas de este riesgo, dadas las características de desempeño de esta área del saber. Realizado el diagnóstico de la flora microbiana de estas dos poblaciones, se pudo concluir que: hay una diferencia significativa entre el porcentaje de individuos afectados por faringitis y parasitosis, con mayor incidencia en las estudiantes del área de la salud; y se comprobó que hay microorganismos que se encuentran presentes en cualquier grupo, como indicativo de la posibilidad de diferentes rutas de infección, en el caso de la presencia de patógenos.
\end{abstract}

Palabras claves: flora bacteriana, profesión, estado de salud.

\begin{abstract}
There are based evidences very well that indicate the validity of the monitoring programs epidemiologist in the prevention and control of the biological risk to avoid the contamination in health atmospheres. Because the workers of the health, are one of the reservorios of the infectious diseases, the C.D.C. and other programs
\end{abstract}


recommend, monitoring, immunization and adoption of, measured of biosegurity and control programs for certain transmissible diseases that they occur in atmospheres where handle health material. One of the objectives of these programs is to protect to those who toil with biological product, against the generated factors of risk, and to fulfill it epidemiologist of individual sayings is due to initiate with the registry of data related to the profile, who allows to design the pertinent strategies for the control of the situation. Therefore, the present study, takes advantage of the investigation to know and to compare the profile epidemiologist of the bacteriology students, in permanent contact with biological risk, in front of moved away students of social work of this risk. Made the diagnosis of microbiota of these two populations, it was possible to be concluded that there is a significant difference between the percentage of individuals affected by faringitis and parasitism, with greater incidence in the students of the area of the health; also it was verified that there are microorganisms that are presents in any group.

Key words: bacterial flora, profession, health condition.

\section{Introducción}

El personal del área de la salud se considera como personal constantemente expuesto al riesgo biológico, ya sea en forma directa como es el caso de las bacteriólogas, los médicos y las enfermeras, o en forma indirecta como es todo el resto de personal que labora en un laboratorio, una clínica, un hospital un consultorio de odontología y otros que se relacionen con atención a pacientes; esta exposición puede ser permanente o temporal debido a que en su quehacer diario, estos profesionales, se enfrentan constantemente a una gran variedad de flora microbiana presente en el ambiente de trabajo y corren el riesgo de infectar o ser infectados, si no cumplen con las normas especiales de seguridad, razón por la cual se utiliza el término bioseguridad para definir y congregar las normas de comportamiento y manejo preventivo del personal de salud frente a microorganismos potencialmente patógenos.

Es así como en 1987 el C.D.C. (Centro para la prevención y control de enfermedades) de Atlanta, como respuesta a la necesidad de disminuir el riesgo de infección de enfermedades ocupacionales enfatizó y publicó las recomendaciones que llamaron "Precauciones Universales". Para su implementación se asume que todo paciente es potencialmente portador de patógenos (1).
A partir de estas consideraciones es indispensable conocer la flora predominante en los individuos que se desempeñan en laboratorios de diagnóstico y en este estudio se seleccionaron las estudiantes de bacteriología de la Universidad Colegio Mayor de Cundinamarca que desarrollan prácticas en diferentes áreas especialmente en microbiologías y parasitología, con el fin de registrar controles relacionados con el cumplimiento de las normas de bioseguridad.

Uno de los parámetros más importantes desde el punto de vista de la Vigilancia Epidemiológica es recolectar información a través del monitoreo del grado de contaminación del ambiente de trabajo, de los individuos que se interrelacionan con los procesos de manejo de fluidos biológicos, desde la recolección de las muestras, el procesamiento de las mismas y el adecuado desecho de todos los productos contaminados y tóxicos (2).

La medida y la evaluación de la microbiota de los individuos que laboran en los laboratorios de diagnóstico son la evidencia indirecta de cómo se están manejando las normas de bioseguridad.

En el presente estudio se evaluó la microbiota de las estudiantes de bacteriología en referencia a la flora bacteriana normal presente en ojos, oídos, nariz y garganta; se determinó la incidencia de parásitos y se 
practicó el reconocimiento del estado de salud de la población, mediante la valoración de signos y síntomas realizada en la consulta médica realizada por las médicas de la IPS de la universidad. En cada uno de los análisis se tuvo en cuenta la validación de los resultados frente a un grupo control, representado en estudiantes que no tenían contacto con material biológico.

Los resultados arrojaron información que indica la mayor exposición y por consiguiente un aumento de la contaminación en las estudiantes que diariamente manejan fluidos corporales con riesgo biológico, ya que demostraron mayor heterogeneidad en las diferentes floras, dentro de las cuales estaban presentes patógenos reconocidos, especialmente en el caso de los parásitos. Así mismo cuando se hizo la correlación entre flora contaminante y el estado de salud, esta arrojó un mayor porcentaje en la incidencia de faringitis y de parasitosis intestinal para las estudiantes de bacteriología frente a las estudiantes de trabajo social que representaron el grupo control.

Como conclusión se pudo determinar que el personal de salud, que manipula fluidos corporales que conllevan el inherente riesgo biológico, y que es imposible de desconocer que se encuentra presente en los profesionales de la salud, presenta una mayor probabilidad de adquirir infecciones, situación que se puede controlar en medida que se apliquen estrictamente las normas de bioseguridad planteadas como estrategia principal en la implementación de la prevención que cuenta con la implementación de los programas de vigilancia epidemiológica como una de las mejores herramientas de control.

\section{Materiales y métodos}

\section{Tipo de estudio y población.}

El estudio se desarrolló durante el primero y segundo semestre del año 2002 y corresponde a un estudio descriptivo, comparativo, retrospectivo, para caracterizar la flora presente en grupos representa- dos por estudiantes de bacteriología de la Facultad de Ciencias de la Salud del Programa de Bacteriología, frente a un grupo integrado por estudiantes de Trabajo Social .

Se realizó análisis retrospectivo para evaluar la presencia de patologías relacionadas con estados infecciosos en cada uno de los grupos. La revisión de las historias clínicas se llevó a cabo en la IPS Manuel Elkin Patarrollo, donde son evaluadas todas las estudiantes de la universidad y se tomaron los datos relacionados con el grupo que participó en el estudio.

\section{Muestra}

Se estudiaron 100 individuos tanto del grupo de Trabajo Social, como del grupo de la muestra, que se escogieron de acuerdo al cumplimiento de los criterios de inclusión planteados.

El grupo de estudio estuvo representado por estudiantes de III, IV, V Y VI semestre del programa de bacteriología que tiene contacto directo con muestras biológicas durante el desarrollo de prácticas en las asignaturas de bacteriologías y parasitología específicamente.

El grupo control estuvo representado por estudiantes de trabajo social que no tienen contacto directo con muestras biológicas en su desempeño diario.

\section{Microbiota de la población en estudio.}

La población estudiada, tanto en el grupo de muestra como en el grupo control fue de 100 individuos en cada uno. Los análisis realizados fueron: cultivos de ojos, nariz, garganta, manos y guantes para evidenciar la microbiota predominante en estas áreas que son las más expuestas; también se realizó coprológico con el fin de conocer la presencia de parásitos en los grupos de estudio. Se aplicó el método estándar para cada una de las determinaciones; en el caso del estudio bacteriológico se practicaron frotis para coloración, se aplicó aislamiento primario, aislamiento secundario, diferenciación bioquímica y pruebas de antibiograma en los casos en que se recuperaron 
patógenos. La valoración de manos y guantes daban razón del oportuno y adecuado manejo de los elementos de barrera.

Para el análisis de los parásitos se practicó valoración microscópica a muestras directas y se aplicó método de concentración para la valoración de huevos.

Los análisis se realizaron durante las clases prácticas para el grupo de bacteriología y para el grupo de Trabajo Social se citaron en sesiones en la mañana, debido a que el estudio es descriptivo y representa el estado de las situaciones en un momento casual.

\section{Reconocimiento del estado de salud de la población.}

Se realizó una revisión de las tasas de morbilidad de las estudiantes de bacteriología y de las estudiantes de trabajo social; la evaluación se hizo durante los periodos de 2002 primero y segundo semestre en referencia a patologías relacionadas con la contaminación microbiana. Se determinó la morbilidad atendida por cuanto se revisaron las historias clínicas que se encuentran en la IPS donde se presta servicio médico a las estudiantes.

\section{Resultados}

\section{Flora de ojos.}

El estudio de conjuntiva realizado al grupo de trabajo social, reportó $80 \%$ de cultivos negativos y $20 \%$ con presencia de Staphilococcus epidermidis, microorganismo considerado habitante normal.

Los cultivos de conjuntiva practicados a las estudiantes de bacteriología reportaron 4\% de muestras negativas para microorganismos, $20 \%$ con presencia de Staphilococcus epidermidis y $70 \%$ con presencia de Streptococcus viridans, bacteria que también se considera habitante normal.

\section{Flora microbiana de nariz.}

Los cultivos de nariz realizados a estudiantes de trabajo social, mostraron presencia de Streptococcus viridans en $80 \%$ de los análisis realizados, $12 \%$ pre- sentaron Staphilococcus epidermidis y $8 \%$ de muestras fueron reportadas como negativas.

En el grupo de bacteriología se reportaron $24 \%$ de cultivos negativos, $20 \%$ de Staphilococcus sp, $46 \%$ de Streptococcus viridans, 9\% deStreptococcus no hemolítico y $1 \%$ de Klebsiella sp.

\section{Flora microbiana de garganta.}

En los cultivos de faringe practicados a las estudiantes de trabajo social se reportaron $80 \%$ de Staphilococcus epidermidis, 5\% de Streptococcus B hemolitico, 10\% de Staphilococcus aureus, 2\% de Streptococcus pneumoniae y 3\% de Streptococcus no hemolítico.

En las muestras de faringe analizadas para las estudiantes de bacteriología se reportaron $20 \%$ de cultivos positivos para Staphilococcus aureus, 10\% positivos para Streptococcus B hemolítico, 30\% para Streptococcus viridans, 25\% para Streptococcus no hemolitico y $15 \%$ para Staphilococcus epidermidis.

En la Figura 1 se presentan los porcentajes que relacionan la flora patógena frente a la flora no patógena encontrada en las muestras de garganta tomadas a las estudiantes de trabajo social.

En la Figura 2 se presentan los porcentajes que relacionan la flora patógena frente a la flora no patógena encontrada en las muestras de garganta de las estudiantes de bacteriología.

\section{Valoración de título de Antiestreptolisinas (ASTOS).}

La prueba de ASTOS practicada a los dos grupos presentaron una positividad del $20 \%$ en las estudiantes de trabajo social frente a $8 \%$ de positivos para las estudiantes de bacteriología.

\section{Flora microbiana de manos y guantes.}

El resultado de los cultivos de manos para las estudiantes de trabajo social reportaron $80 \%$ negativos, 7\% Bacillus subtilis, y 13\% S epidermidis.

www.unicolmayor.edu.co 
Los resultados de los cultivos de manos y guantes de las estudiantes de bacteriología se reportan en la tabla 1 donde se evidencia las presencia de patógeno espalmente entéricos.

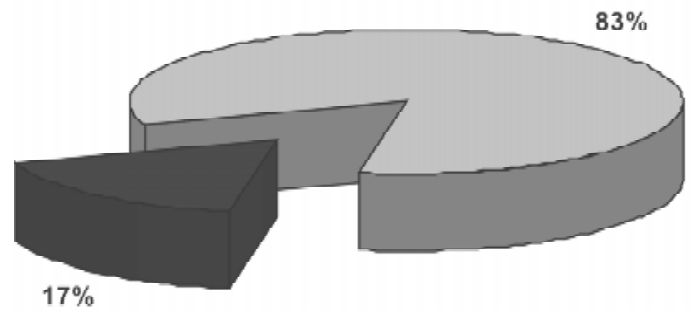

$$
\text { घPatógenos } \square \text { No Patígenos }
$$

Figura 1. Microorganismos de garganta. Grupo de Trabajo social. En la gráfica se aprecian los porcentajes de bacterias encontradas en muestras de faringe donde se diferencia el \% de patógenos y no patógenos encontrados en las estudiantes de trabajo social

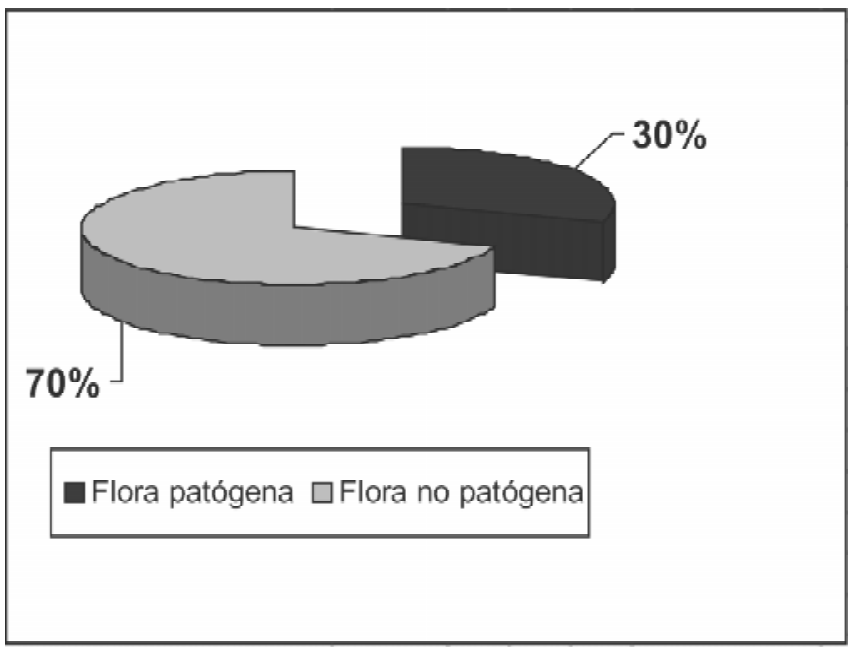

Figura 2. Microorganismos de garganta. Grupo de bacteriología. En la gráfica se aprecian los porcentajes de bacterias encontradas en muestras de faringe donde se diferencia el \% de patógenos y no patógenos encontrado en las estudiantes de bacteriología

\section{Incidencia de parásitos}

Se practicó valoración de parásitos a un grupo de estudiantes de trabajo social que no están expuestas a riesgo porque no manipulan muestras de coprológicos

Se encontró un $8 \%$ de parásitos presentes en las estudiantes de bacteriología.
En referencia a los análisis realizados para evaluar la aplicación adecuada de los elementos de barrera como son los guantes se encontraron bacterias patógenas tanto en las manos como en los guantes de las estudiantes de bacteriología, como se demuestra en la Tabla 1.

\section{Reconocimiento del estado de salud de la población}

Se practicó la revisión de los datos de morbilidad de estudiantes de Bacteriología que representa el grupo expuesto a riesgo biológico y se tomó como referencia de población que no está expuesta a riesgo biológico, las estudiantes de Trabajo Social.

Los resultados del grupo control se presentan en las tablas 1,2 y 3.

Cuando se comparan las frecuencias de aparición de procesos infecciosos entre las dos facultades queda claramente establecida la mayor incidencia de patología infecciosa en las estudiantes del programa de Bacteriología frente a las estudiantes del programa de Trabajo Social; es el caso de entidades de común aparición como la Faringitis que presenta en Bacteriología un $57 \%$ en el acumulado mientras que en trabajo social solo hay un $34 \%$; así mismo sucede con el parasitismo con un $15.9 \%$ para Bacteriología, contra $8.5 \%$ para Trabajo Social.

\section{Discusión.}

En la concepción moderna de atención en salud es necesario tener en cuenta tanto la atención al medio ambiente como la atención a las personas; en relación al medio ambiente la atención se centra en el control de la contaminación en los diferentes espacios físicos en relación con el aire, los equipos, las instalaciones con control de mesones, paredes, pisos, los desechos y otros, esta situación en el caso de los laboratorios de diagnóstico.

En relación con los individuos, en el presente estudio se relaciona con los profesionales de bacteriología que son quienes están en mayor contacto con las muestras

www.unicolmayor.edu.co 
Tabla 1. Bacterias Gram negativas presentes en manos y guantes de estudiantes de bacteriología.

\begin{tabular}{cccc}
\hline Manos & Porcentaje & Guantes & Porcentaje \\
\hline Enterobacter sakasaki & $10.71 \%$ & Citrobacter diversus & $21.42 \%$ \\
Enterobacter cloacae & $10.71 \%$ & Alcaligenes fecalis & $7.14 \%$ \\
Hafnia alvei & $7.14 \%$ & Serratia marcescens & $7.14 \%$ \\
Enterobacter aerogenes & $10.71 \%$ & Proteus mirabilis & $17.85 \%$ \\
Shiguella sonnei & $7.14 \%$ & & \\
\hline
\end{tabular}

Tabla 2. Porcentaje de incidencia de patologías presentadas por estudiantes de Trabajo Social

\begin{tabular}{ccccc}
\hline $\mathbf{N}^{\circ}$ de orden & $\begin{array}{c}\text { Código de la } \\
\text { enfermedad }\end{array}$ & $\begin{array}{c}\text { Nombre de la } \\
\text { patología }\end{array}$ & Frecuencia & Porcentaje \\
\hline 1 & 6793 & Sano & 54 & $22.5-88.8$ \\
5 & 129 & Parasitismo & 10 & $3.3-8.5$ \\
2 & 462 & Faringitis aguda & 14 & $4.6-34.4$ \\
& 4658 & Infecciones & 11 & $3.2-27.6$ \\
& & respiratorias & & $3.6-47.2$ \\
& 536 & Trastornos & 11 & \\
\hline
\end{tabular}

Tabla 3. Porcentaje de incidencia de patologías presentadas por estudiantes de Bacteriología

\begin{tabular}{cccccc}
\hline $\mathbf{N}^{\circ}$ de orden & $\begin{array}{c}\text { Código de la } \\
\text { enfermedad }\end{array}$ & $\begin{array}{c}\text { Nombre de la } \\
\text { patología }\end{array}$ & Frecuencia & Porcentaje & Acumulado \\
\hline 2 & 686 & Infección piel & 18 & $4.1 \%$ & 32.1 \\
3 & 595 & $\begin{array}{c}\text { Cistitis } \\
\text { Parasitosis }\end{array}$ & 17.75 & $4.0 \%$ & 32 \\
4 & 129 & intestinal & 15.9 & $1.6 \%$ & 15.9 \\
5 & 007 & Protozoario & 14 & $2.3 \%$ & 13.9 \\
1 & 462 & Faringitis & 22.5 & $3.8 \%$ & 57 \\
\hline
\end{tabular}


biológicas porque son precisamente estos profesionales los encargados de la manipulación de todas las muestras compuestas de fluidos biológicos que se consideran por principio como contaminantes y conllevan a exponer a las personas a riesgo biológico. Se ha determinado que la única forma de lograr un trabajo seguro y de poder contrarrestar los factores de riesgo es la aplicación de la prevención entendida como la óptima aplicación de la bioseguridad (1).

La labor preventiva frente a la presencia de riesgo biológico no se reduce solamente a las acciones de desinfección, descontaminación, limpieza, esterilización y antisepsia, sino que requiere de acciones educativas, investigativas y evaluativos, que deben ser implementadas de forma continua para el aseguramiento de la calidad en el proceso de control de la diseminación de la contaminación y así garantizar un proceso libre de accidentes y minimización del riesgo (3).

La investigación constituye uno de los elementos fundamentales para la aplicación de un plan de prevención, porque permite que mediante la tecnología en los laboratorios se pueda monitorear la flora presente en los diferentes elementos y así se puedan adelantar importantes trabajos de seguimiento de casos, de determinación de gérmenes ambientales, de contaminación de instalaciones, de equipos y de materiales. La actividad de investigación sin lugar a dudas representa el mejor sello de garantía de calidad en las instituciones de salud (2).

Otro elemento indispensable está relacionado con la evaluación constante que permite que se tomen correctivos oportunos mediante el diseño de protocolos, normas, procedimientos, directrices generales y actualización de métodos. El costo de la infección es muy alto en toda entidad y a todo nivel porque si se relaciona la contaminación que se presente en una institución hospitalaria, esta situación requiere medicamentos, análisis de laboratorio, imágenes diagnósticas, lucro cesante y otros, siendo el mayor problema el poner en peligro la integridad de los individuos (3).
El presente estudio se realiza en cumplimiento de uno de los aspectos fundamentales del modelo de vigilancia epidemiológica planteado para el control de la contaminación biológica en los laboratorios de diagnóstico en el programa de bacteriología de la facultad de Ciencias de la Salud y que tiene como principio conocer las actividades biológicas que explican la causalidad de las infecciones como producto de la interacción de múltiples factores entre los que se cuentan: el agente microbiológico, el reservorio es decir el lugar donde residen los microorganismos, el intestino, la faringe, la piel, los orificios naturales de las personas infectadas o de las que se presentan como portadores sanos y es precisamente esta población la de interés particular en la presente investigación, donde se pudo demostrar claramente la mayor exposición a los agentes patógenos para los individuos que manejan muestras biológicas (4).

En los análisis practicados a los individuos quedó demostrado la mayor incidencia de patógenos en los estudiantes de bacteriología, situación que está acorde con los índices de morbilidad analizados en comparación con las estudiantes de trabajo social.

En referencia a los microorganismos presentes en manos y guantes se encontraron gran variedad de gérmenes muchos de estos patógenos como se observa en la tabla 1 estos resultados obtenidos son indicativos de deficiencias en la aplicación de las normas de bioseguridad y debilidad en el lavado de manos (5).

La valoración de parásitos mostró una diferencia significativa entre el grupo de muestra y el grupo control. La evaluación de las infecciones respiratorias demostró la incidencia de faringitis con agentes específicos como Staphylococcus y Streptococcus confirmado con pruebas de ASTOS que confirmaron la presencia de patógenos como Streptococcus $B$ hemolítico y Streptococcus pneumoniae en el grupo de estudio (6).

Hay que tener en cuenta que los microorganismos patógenos de garganta tienen muchas vías de contaminación no solamente el contacto con muestras biológicas, situación que se ratifica con el análisis de 
ASTOS que mostró mayor incidencia de positividad en el grupo control, situación que demuestra la presencia de contaminación en estos individuos con estreptococos patógenos.

En consecuencia se puede concluir que el diseño de la recolección de datos a cerca de la microbiota presente en los individuos, establecido en el presente estudio está de acuerdo con parámetros indicados en el estudio epidemiológico de la subdirección de la red hospitalaria de Vigilancia epidemiológica Rhove (file: $\mathrm{Ai} /$ rhohe vigilancia qpidemiol.him) donde se recomiendan los pasos indispensables a seguir para la implementación de un programa de control de infecciones e indican claramente coleccionar una información oportuna y adecuada de las enfermedades, riesgo y daños de salud que se presentan en las unidades de servicios de salud para documentar el panorama epidemiológico y así poder orientar adecuadamente los programas de control (5)(6).

Un aspecto importante de tener en cuenta y desarrollar adecuadamente son los estudios que conduzcan a definir los patrones epidemiológicos de las infecciones nosocomiales, así mismo, es indispensable conocer la ecología microbiana del sitio de estudio y revisar periódicamente la situación, para conocer que cambios se están dando en esa flora, porque la aparición de patógenos importantes serán representativos de cambios actitudinales frente a la aplicación estricta de las normas de bioseguridad (7).
Del cuidado que se tenga en relación a la obtención de datos oportunos para determinar las acciones a seguir frente a las diferencias en las floras microbianas presentes, dependerá en gran medida el control del riesgo de diseminación de la contaminación. Es una necesidad sentida en la actualidad insistir constantemente en las facultades de atención en salud en el adecuado manejo del autocuidado, de la aplicación adecuada de las normas de bioseguridad y la aplicación de la prevención y control del riesgo biológico, porque hemos tenido que vivenciar precisamente en estos días, situaciones muy difíciles en varios centros hospitalarios en relación con estas situaciones que han cobrado varias vidas.

\section{Referencias}

1. Arroyabe M. Infección Intrahospitalaria. Prevención y control. Hospital Universitario San Vicente de Paul. 3. Edición. Medellín, Colombia;1999.

2. Malagón G. Infecciones hospitalarias. 2 Edición. Bogotá: Panamericana; 1999.

3. Chow A. Principles and practice of infection diseases, 2002; p 689-699.

4. Whitehead J. Manual de Bioseguridad en el Laboratorio. Londres: Servicio de laboratorio de Salud Pública, 2002.

5. Fernández L, De la Cruz C. Riesgo ocupacional y Medidas de Seguridad en los laboratorios médicos. Instituto de Medicina Tropical Kouri. CEPIS 2002. Disponible En : URL: http:// www.cepis.ops-oms.org/eswww/fulltest/repino61/rboomslm/ rbooms.htm.

6. Jawest E Microbiología Médica. Editorial Manual Moderno; 1996.

7. Campuzano S, González A. Bioseguridad en la consulta de oftalmología y de optometría. 1 Edición. Universidad Colegio Mayor de Cundinamarca. 2005. 